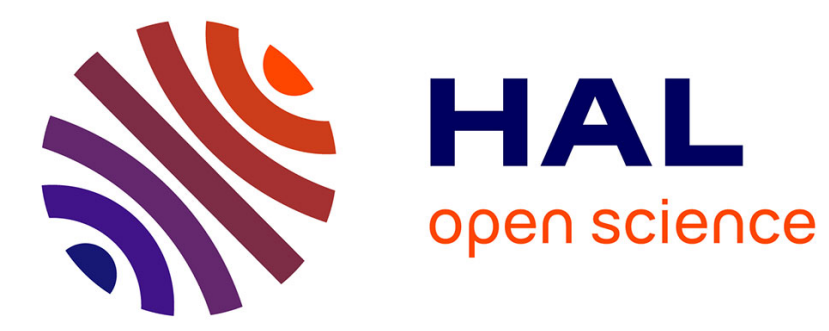

\title{
Interaction Energy in some Phenomenological Modelings of Phase Transitions in Crystalline Solids \\ Christian Licht
}

\section{To cite this version:}

Christian Licht. Interaction Energy in some Phenomenological Modelings of Phase Transitions in Crystalline Solids. Michel Frémond; Franco Maceri. Novel Approaches in Civil Engineering, 225-232, Springer, 2004, 978-3-540-41836-8. 10.1007/978-3-540-45287-4_18. hal-03342797

\section{HAL Id: hal-03342797 https://hal.science/hal-03342797}

Submitted on 14 Sep 2021

HAL is a multi-disciplinary open access archive for the deposit and dissemination of scientific research documents, whether they are published or not. The documents may come from teaching and research institutions in France or abroad, or from public or private research centers.
L'archive ouverte pluridisciplinaire HAL, est destinée au dépôt et à la diffusion de documents scientifiques de niveau recherche, publiés ou non, émanant des établissements d'enseignement et de recherche français ou étrangers, des laboratoires publics ou privés. 


\section{Interaction Energy in some Phenomenological Modelings of Phase Transitions in Crystalline Solids}

\section{Christian Licht}

Laboratoire de Mécanique et Génie Civil, cc 048, Université Montpellier II, F-34095 Montpellier Cedex 5, France

Abstract. Some phenomenological and macroscopic modelings of solid/solid phase transitions involve a so-called interaction term which may imply the nonconvexity of the strain energy function. Nevertheless, it is shown that, if this term is not too large, these modelings are valuable and account for some experimental facts.

\section{Introduction}

I will present some remarks on a category of phenomenological modelings of phase transition in crystalline solids. These modelings involve a so-called interaction term which may imply the nonconvexity of the strain energy density function of the medium. This lack of sacrosanct convexity generates some controversy, warning, or nonuse (see some comments in [2], [8], [9], [13], [19]).

I will show that, if this term is not too large, the modeling is valuable. Certainly, the stored energy density function is not convex but quasiconvex and, consequently, inherits a double-well like structure which may account for some experiments.

From a technical, say mathematical, standpoint, this study is nothing but a slight variant of the beautiful papers [11], [12]... Nevertheless, I hope it may interest people concerned with the modeling of shape memory alloys.

\section{Setting the problem}

First, the phenomenological modelings, that I will discuss, describe the state of the medium by a triple $(\epsilon, x, T) ; \epsilon, T$ stand respectively for the linearized strain tensor and the temperature, while $0 \leq x \leq 1$ denotes a volume fraction. Since a volume fraction is put forward, note that this modeling operates at a large, say macroscopic, scale. Moreover, $x$ being a scalar, I will consider only two phases; indeed considering more phases implies some substantial difficulties (except in obvious symmetry related phases). Thus this modeling concerns the detwining of two variants of martensite (martensite may present two variants in the case of soda, neodynium pentaphosphate, wolfram, ... ) or is appropriate to polycristalline alloys where the variants of martensite 
are not told apart in the austenite-martensite mixture. Next, a free energy density function is defined by a function $W$ of the state variables. At last, the phase transitions are assumed nondissipative, which seems rather realistic, so that the medium has a thermoelastic behavior with a stored energy density function

$$
\bar{W}(\epsilon, T)=\operatorname{Inf}\{W(\epsilon, T, x), 0 \leq x \leq 1\}
$$

Of course, the whole point is the choice of $W$; at least two approaches lead to the category of modelings that I consider. The first approach, which models the medium as a generalized standard medium [10], splits the freeenergy into three terms:

$$
W(\epsilon, x, T)=W^{e}(\epsilon-x a, T)+x W^{c h}(T)+W^{\phi}(x, T)
$$

The first term is the thermoelastic one, $x a$ being the phase transformation part of the total strain $\epsilon$, while the second one concerns the latent heat exchange. The last one is the stored part due to internal changes, it is affected by the interactions between phases thus it has to be minimal and equal to 0 at $x=0$ or $x=1$. Obviously $x \in[0,1] \longmapsto x(1-x)$ is a simple and smooth function which satisfies the previous conditions, so a proposal for $W^{\phi}$ is:

$$
W^{\phi}(x, T)=\phi(T) x(1-x), \phi \geq 0
$$

Note that in [14], [15], [16], it is reported that $x(1-x)$ is proportional to the expectation value of the interfacial area, if the phases are randomly distributed over the specimen ... Moreover, this modeling implicitly assumes that the elastic coefficients of the phase are equal, so this modeling confines to detwining.

The second approach regards the medium as a mixture such that the free energy may be a $x$-weighted combination of the energies $W^{i}, i=1,2$, of each phase. But, referring to homogenization, subtracting a term $W^{c}$, minimal and equal to 0 at $x=0$ or $x=1$, yields a better estimate:

$$
W(\epsilon, x, T)=x W^{1}(\epsilon, T)+(1-x) W^{2}(\epsilon, T)-W^{c}(x, T)
$$

Once again, a convenient choice is

$$
W^{c}(x, T)=c(T) x(1-x), c \geq 0
$$

Note that this approach does not assume the identity of the elastic laws of the two phases. From the mixture standpoint, another approach, which will be detailed in remark 3.1, leads to an expression like (1) in the case of two linearly elastic phases with identical elastic moduli. More deeply, this last assumption, which will stand for all the sequel, implies the identity of the two approaches. More precisely, I assume 


$$
W^{i}(\epsilon, T)=1 / 2 \alpha\left(\varepsilon-a_{i}(T)\right) \cdot\left(\varepsilon-a_{i}(T)\right)+w_{i} \quad, i=1,2
$$

where $a_{i}$ is the stress-free strain of the $\mathrm{i}$-th phase, $w_{i}$ the associated minimum energy and $\alpha$ the tensor of elastic moduli, a positive symmetric linear map on the space $S^{3}$ of symmetric matrices. Then the obvious identity

$$
\begin{aligned}
& x W^{1}(\epsilon, T)+(1-x) W^{2}(\epsilon, T)-c(T) x(1-x)= \\
& 1 / 2 \alpha(\epsilon-\bar{a}(T)) \cdot(\epsilon-\bar{a}(T))+\bar{w}(x, T)+\phi(T) x(1-x)
\end{aligned}
$$

where

$$
\begin{gathered}
\bar{a}=x a_{1}+(1-x) a_{2} \quad, \quad \bar{w}=x w_{1}+(1-x) w_{2} \\
\phi=1 / 2 \alpha a \cdot a-c \quad, a=a_{2}-a_{1}
\end{gathered}
$$

yields a single modeling where two equivalent expressions of the free energy are given by the two members of this identity!

It is not my purpose to list all the authors who, specifically or vaguely, proposed or discussed this modeling. Due to the many studies devoted to Shape Memory Alloys, it is impossible to be exhaustive. Generally, some information may be found in [2], [6]-[9], [13]-[17], [19] and in the references therein. My main thrust is to decide on the value of a nondissipative modeling where the free energy and the strain energy functions are :

$$
\begin{aligned}
& W_{\phi}(\epsilon, x, T)=1 / 2 \alpha(\epsilon-\bar{a}(T)) \cdot(\epsilon-\bar{a}(T))+\bar{w}(T)+\phi(T) x(1-x) \\
& =x W^{1}(\epsilon, T)+(1-x) W^{2}(\epsilon, T)-c(T) x(1-x), c=1 / 2 \alpha a . a-\phi
\end{aligned}
$$

and

$$
\bar{W}_{\phi}(\epsilon, T)=\operatorname{Inf}\left\{W_{\phi}(\epsilon, T, x), 0 \leq x \leq 1\right\}
$$

Clearly, from the first expression, $\phi$ non positive implies that $W_{\phi}$ is a convex function of the couple $(\epsilon, x)$ and, consequently, that $\bar{W}_{\phi}$ is a convex function of $\epsilon$. But, as previously noted, $\phi$ negative does not seem plausible! ... Moreover, it is not difficult to see that $\bar{W}_{\phi}$ is a convex function in $\epsilon$ if $\phi$ is positive. In fact, it is not a disaster. I will show that if $\phi$ is positive, but not too large, the modeling is valuable... In the following technical discussions, temperature will only play a role of parameter, thus temperature dependence is suppressed hereafter. 


\section{3 (Quasi)convexity properties of $\bar{W}_{\phi}$}

A mechanical interpretation of the mathematical theory of quasiconvexification [5], [18] may be : "at the macroscopic scale the behavior of a hyperelastic material is not governed by the true stored energy function but by an apparent one : its quasiconvexification, which is the infimum of those average energies that can be attained in deformations with a given average". Hence a first criterion of a good macroscopic modeling is the quasiconvexity property of the stored energy density function $\bar{W}$ which, in our case, may be read as :

$$
\bar{W}(E)=\operatorname{Inf}\left\{1 /|U| \int_{U} \bar{W}(E+\epsilon(u)) d y ; u(y)=0 \text { on } \partial U\right\}, \forall E \in S^{3}
$$

where the choice of the domain $U$ is unimportant. Note that convexity implies quasiconvexity but the converse is generally false. Here are some (quasi)convexity properties of the strain energy function $\bar{W}_{\phi}$ :

\section{Theorem 3.1. Let}

$h=\operatorname{Min}\left\{1 / 2 \alpha(z-a) \cdot(z-a) ; z=k \otimes v+v \otimes k, k, v \in R^{3}\right\} \in[0,1 / 2 \alpha a \cdot a]$

then

$\phi \leq 0$ implies $\bar{W}_{\phi}$ is convex,

$h>0$ and $0 \leq \phi \leq h$ implies $\bar{W}_{\phi}$ is not convex but quasiconvex,

$h<\phi$ implies $\bar{W}_{\phi}$ is not quasiconvex.

Moreover, $\bar{W}_{\phi}$ equals $\bar{W}_{1 / 2 \alpha a \cdot a}$ if $\phi \geq 1 / 2 \alpha a \cdot a$.

As noted in [11], $h$ differs from 0 if and only if the full transformation strain $a$ is not a symmetrized tensor product, in other words if the two stress-free strains are not compatible. Thus if $h>0$, it can be claimed that the modeling with $\phi \in[0, h]$, is a "good" macroscopic modeling, whereas only $\phi=0$ yields a good modeling if $h=0$ !

Proof of theorem 3.1. Several arguments of [11] pp 201-203 are used without giving details. First, in the definition of quasiconvexity, a unit cell $Y$ and periodicity conditions may be used in place of $U$ and Dirichlet conditions. Standard results on mesurable selections imply :

$$
\begin{aligned}
I & :=\operatorname{Inf}\left\{\int_{Y} \bar{W}_{\phi}(E+\epsilon(u)) d y ; u \in C_{p e r}^{1}(\bar{Y})\right\} \\
& =\operatorname{Inf}\left\{\int_{Y} W_{\phi}(E+\epsilon(u), \theta(y)) d y ; u \in C_{p e r}^{1}(\bar{Y}), \theta \in B(Y)\right\}
\end{aligned}
$$


where $B(Y):=\left\{\theta \in L^{\infty}(Y) ; 0 \leq \theta \leq 1\right\}$. An elementary calculation using the second expression of $W_{\phi}$ in (4) gives

$$
\begin{gathered}
I=\operatorname{Inf}\left\{m W^{1}(E)+(1-m) W^{2}(E)-c m+J(m) ; 0 \leq m \leq 1\right\}, \\
J(m)=\operatorname{Inf}\left\{\int_{Y} c \theta^{2}+1 / 2 \alpha \epsilon(u) \cdot \epsilon(u)+\theta \alpha \epsilon(u) \cdot a ;\right. \\
\left.u \in C_{p e r}^{1}(\bar{Y}), \theta \in B(Y), \int_{Y} \theta=m\right\} .
\end{gathered}
$$

As in [11] p. 202, Fourier analysis yields

$$
\begin{aligned}
& J(m) \geq \operatorname{Inf}\left\{\mathrm{cm}^{2}+(h-\phi) \sum_{k \neq 0} \hat{\theta}(k)^{2} ;\right. \\
& \left.y \in Y \mapsto \theta(y)=\sum_{k \in Z^{3}} \hat{\theta}(k) e^{2 i \pi k \cdot y} \in B(Y)\right\}
\end{aligned}
$$

which proves the quasiconvexity of $\bar{W}_{\phi}$ if $0 \leq \phi \leq h$.

Clearly, (4), (5) imply that $\bar{W}_{\phi_{1}} \geq \bar{W}_{\phi 2}$ if $\phi_{1}>\phi_{2}$, and that $\bar{W}_{\phi}=$ $\bar{W}_{1 / 2 \alpha a . a}=\operatorname{Min}\left(W^{1}, W^{2}\right)$ if $\phi \geq 1 / 2 \alpha a \cdot a$. Moreover, it is shown in [11] that the quasiconvexification and the convexification of $\operatorname{Min}\left(W^{1}, W^{2}\right)$ are respectively $\bar{W}_{h}$ and $\bar{W}_{0}$. Thus, $\bar{W}_{\phi}$ is not convex if $0<\phi$ and is not quasiconvex if $h<\phi$, because quasiconvexification and convexification preserve the order.

Remark 3.1. A rough way to define the strain energy function of a mixture of two phases described by the energy density functions $W^{i}$, in proportions $x$ and $(1-x)$, is to consider

$$
W_{a}^{m i x}(\epsilon, x)=\operatorname{Inf}\left\{x W^{1}\left(\epsilon^{1}\right)+(1-x) W^{2}\left(\epsilon^{2}\right) ; x \epsilon^{1}+(1-x) \epsilon^{2}=\epsilon\right\}
$$

With $W^{i}$ as in (3), it is shown in [11] that

$$
W_{a}^{m i x}(\epsilon, x)=W_{0}(\epsilon, x)=1 / 2 \alpha(\epsilon-\bar{a}(x)) \cdot(\epsilon-\bar{a}(x))+\bar{w}(x)
$$

Thus, as announced previously, in order to account for interactions at a macroscopic level, some modelings ([6]-[9], [13]-[16], [19]) add a term depending on $x$, vanishing at $x=0,1$. Their common proposal is $\phi x(1-x)$, hence they get $W_{\phi}$ as free energy density function for the medium! However, the previous definition $W_{a}^{\text {mix }}$ presupposes constant and compatible strains in each phase, which is open to criticism. A better definition is

$$
\begin{gathered}
W_{b}^{m i x}(\varepsilon, x)=\operatorname{Inf}\left\{1 /|Y| \int_{Y} \chi_{x}(y) W^{1}(\varepsilon(u))+\left(1-\chi_{x}(y)\right) W^{2}(\varepsilon(u)) d y\right. \\
\left.\chi_{x} \text { characteristic function } \int_{Y} \chi_{x}(y) d y=x|Y|, u(y)=\varepsilon y \text { on } \partial Y\right\}
\end{gathered}
$$


It is proved in [11] that $W_{b}^{m i x}=W_{h}$. Hence, adding a macroscopical term of interaction $I x(1-x)$ yields a free energy equal to $W_{h+I}$. Then the quasiconvexity criterion of good modeling implies that $I$ must be non positive. This sign, which is the opposite of what is widely used in the literature, corresponds exactly to what in [4] is termed, through microscopic considerations, mismatch energy.

\section{Double-well like structure of $\bar{W}_{\phi}$ and experiments}

From now on, I confine to the more interesting case $0<\phi \leq h$ and will show that the lack of convexity of $\bar{W}_{\phi}$ accounts for some experimental results, which also guarantees a good modeling. Let

$$
w=w_{1}-w_{2}, H_{1}=\left\{e \in S^{3} ; \alpha\left(e-a_{1}\right) \cdot a=w\right\}
$$

Some elementary manipulations, as in [11] pp. 205-207, provide the following geometric properties of the graph of $\bar{W}_{\phi}$ :

Proposition 4.1. $\bar{W}_{\phi}$ is piecewise quadratic and differentiable on $\mathrm{S}^{3}$. If $|w|>\phi, \bar{W}_{\phi}$ has only one absolute minimizer: $a_{1}$ (resp. $a_{2}$ ) if $w>\phi$ (resp. $w<-\phi)$, and no other relative minimizers. If $|w| \leq \phi, \bar{W}_{\phi}$ has exactly two relative minimizers $a_{1}$ and $a_{2}: a_{1}$ (resp. $a_{2}$ ) is an absolute minimizer if and only if $w \geq 0$ (resp. $w \leq 0)$.

Proposition 4.2. For each $e_{1}$ of $H_{1}$, the function $\bar{W}_{\phi}-\Sigma_{1} \cdot$, with $\Sigma_{1}=\alpha\left(e_{1}-a_{1}\right)$, has exactly two absolute minimizers $e_{1}$ and $e_{2}=e_{1}+a$.

Since $\bar{W}_{\phi}$ is differentiable, the previous properties can be interpreted in terms of stress and strain. First, it appears that along all straight paths $\epsilon_{\Delta}$ in the strain space $S^{3}, t \in R \longmapsto \epsilon_{\Delta}(t)=\epsilon_{0}+t a$, parallel to $a$, the derivative of the energy $\bar{W}_{\phi}\left(\epsilon_{\Delta}(t)\right)$ is not monotone. In other words, "the stress-strain relation is not monotone along $\epsilon_{\Delta}$ ". Thus it should be of interest to do such strain controlled tests which must be isothermal and biaxial (in this direction see [6], [14], [15]). These hard device tests seem difficult to do, conversely it is easier to do isothermal and biaxial loading experiments [3]. Leaving hysteresis considerations aside, it seems clear that two different equilibrium configurations may occur when a specimen of shape memory alloy is subjected to certain uniform biaxial extension loadings. The present modeling may account for this fact. If $\Omega$ is a reference configuration of the specimen and $n$ the unit outward normal to $\partial \Omega$, the total energy read as

$$
I_{\Sigma}(v)=\int_{\Omega} \bar{W}_{\phi}(\epsilon(v)) d x-\int_{\partial \Omega} \Sigma n . v d s=\int_{\Omega} \bar{W}_{\phi}(\epsilon(v))-\Sigma \cdot \epsilon(v) d x
$$

The previous propositions make it possible to use the arguments in [12] pp $82-83$ and to prove the following metastability theorem :

Theorem 5.1. For every $e_{1} \in H_{1}$, there exists $\delta>0$ such that for every $\Sigma$ in a ball in $S^{3}$ of center $\Sigma_{1}=\alpha\left(e_{1}-a_{1}\right)$ and radious $\delta$, the 
functional $I_{\Sigma}$ has two distinct $L^{1}$-local minimizers (one of them being an absolute minimizer in $\left.H^{1}(\Omega)^{3}\right)$. As $\Sigma$ goes to $\Sigma_{1}$, they converge strongly in $L^{1}$ towards " $x \longmapsto e_{1} x "$ and " $x \longmapsto\left(e_{1}+a\right) x "$, absolute minimizers of $I_{\Sigma_{1}}$ in $H^{1}(\Omega)^{3}$.

The $L^{1}$ topology is rough : in $L^{1}$ neighborhoods the displacements are near in the mean, but their strains may be very remote. These local, but non absolute, minimizers may be observed, they correspond to metastable equilibrium configurations : experimentalists [3] report that specimen can undergo large deformations from an equilibrium configuration to another one only by slightly hitting the strings of the device or through a small disturbance in the room. A similar metastability analysis, in the more difficult framework of finite strains, may be found in [1].

\section{Conclusion}

I believe that all the foregoing arguments clearly show that the considered modeling is coherent and able to account some phenomena involved by phase transitions in crystalline solids. According to remark 3.1, a nice introduction of this phenomenological and macroscopic modeling is adding a rational micro-macro definition of the energy function of a mixture to a woolly termed function, $I x(1-x), I \leq 0$, of the volume fraction $x$ of one phase. It should be interesting to derive this last term through a rational scale transition that, for instance, takes into account micro-scale surface interactions.

Eventually, I thank my friend C. Lexcellent who told me about some pertinent earlier works.

\section{References}

1. Ball J M, James R D (1995) Local minimizers and phase transformations. Proceedings of the ICIAM

2. Chrysochoos A (1998) Vers une reformulation des transitions de phase du premier ordre. Rev. Roum. Sci. Techn. - Méc. Appl., Tome 43 no 3:283-293

3. Chu C (1993) Hysteresis and Microstructures: A study of Biaxial Loading on Compound Twins of Copper-Aluminium-Nickel Single Crystals. Dissertation , University of Minesota.

4. Chu C, James R D (1993) Biaxial loading experiments on Cu-Al-Ni single Crystals. In: Experiments in Smart Materials and Structure, ASME, AMD vol 181, pp 61-69

5. Dacorogna B (1989) Direct Methods in the Calculus of Variations, Applied Mathematical Sciences, vol 78, Springer, Berlin Heidelberg New York

6. Fu S, Huo Y, Müller I (1993) Thermodynamics of Pseudoelasticity - An Analytical Approach. Acta Mech. 991

7. Fedelich B, Zanzotto G. (1991) One-dimensional quasistatic nonisothermal evolution of shape-memory material inside the hysteresis loop. Continuum Mech. Thermodyn. 3:251-276 
8. Goo B C, Lexcellent C (1997) Micromechanics-based Modeling of Two-way memory effect of a single crystalline shape-memory alloy. Acta Mater vol 45 no 2:727-737

9. Huo Y, Müller I (1993) Non-Equilibrium Thermodynamics of Pseudoelasticity. Continuum Mech. Thermodyn. 5

10. Halphen B, Nguyen Q S (1975) Sur les matériaux standards généralisés. Journal de Mécanique 14:39-63

11. Kohn R V (1991) The relaxation of a double-well energy. Continuum Mech. Thermodyn. 3:193-236

12. Kohn R V, Sternberg P (1989) Local minimizers and singular perturbations. Proceedings of the Royal Society of Edinburgh 111A:69-84

13. Lexcellent Ch, Boubakar M L (200) About the thermomechanical modelling of shape memory alloys. In Continuum Thermomechanics the Art and Science of Modelling Material Behaviour, Paul Germain's Anniversary. Kluwer, London, pp 225-236

14. Müller I (1989) On the size of hysteresis in pseudo-elasticity. Continuum Mech. Thermodyn. 1:125-142

15. Müller I, Xu H (1991) On the pseudo-elastic hysteresis. Acta Metall. 39:263-271

16. Müller I. (1996) Six lectures on Shape Memory Alloys, Technical University Berlin

17. Pagano S, Alart P (1999) Solid-solid phase transition modelling : relaxation procedures, configurational energies and thermomoechanical behaviours. International Journal of Engineering Science, vol 37 no 14:1821-1840

18. Pipkin (1986) Some examples of Crinkles. In: Ericksen J L (eds) Homogenization and Effective Moduli of Materials and Media. IMA vol Math. Appl. 1. Springer, Berlin Heidelberg New York, pp 182-195

19. Raniecki B, Lexcellent C, Tanaka K (1992) Thermodynamic model of pseudoelastic behaviour of shape memory alloys. Arch. Mech. vol 44 no3:261-284 